\title{
Investigating critical genes and gene interaction networks that mediate cyclophosphamide sensitivity in chronic myelogenous leukemia
}

\author{
XIAO HE, YUYING DENG and WEI YUE
}

Blood Transfusion Department, The First People's Hospital of Yancheng City, Yancheng, Jiangsu 224006, P.R. China

Received January 21, 2016; Accepted February 15, 2017

DOI: $10.3892 / \mathrm{mmr} .2017 .6636$

\begin{abstract}
Drug resistance is an obstacle in the treatment of chronic myelogenous leukemia (CML), and is a common reason for treatment failure or disease progression. However, the underlying mechanisms of cyclophosphamide resistance remain poorly defined. In the present study, microarray data concerning cyclophosphamide-sensitive and -resistant chronic myelogenous leukemia cell lines were analyzed. A total of 258 differentially-expressed genes (DEGs) were identified between these two groups, from which 139 DEGs were upregulated and 119 were downregulated. Several candidate genes that were associated with cyclophosphamide resistance were also identified. These DEGs were subsequently classified using Gene Ontology and Kyoto Encyclopedia of Genes and Genomes (KEGG) enrichment pathway analysis. A total of 487 biological processes and 17 KEGG pathways were revealed to be enriched. Furthermore, an interaction network was established to identify the core genes that regulated cyclophosphamide resistance. Signal transducer and activator of transcription 5A (STAT5A), FYN proto-oncogene, Src family tyrosine kinase and spleen associated tyrosine kinase were revealed to be the hub genes in multiple enriched biological processes and signaling pathways, indicating that these were involved in mediating cyclophosphamide sensitivity in CML cells. The expression levels of 5 DEGs were also confirmed in two human CML cell lines (K-562 and KU812) by reverse transcription-quantitative polymerase chain reaction. Furthermore, selective knockdown of STAT5A and S100 calcium binding protein A4 (S100A4) recovered cyclophosphamide sensitivity in K-562 cells, suggesting their involvement in drug resistance. The present study identified several potential genes and pathways contributing to cyclophosphamide resistance, and confirmed the involvement of STAT5A and S100A4 in drug
\end{abstract}

Correspondence to: Dr Xiao He, Blood Transfusion Department, The First People's Hospital of Yancheng City, 166 West Yulong Road, Yancheng, Jiangsu 224006, P.R. China

E-mail: hexiao_js@163.com

Key words: chronic myelogenous leukemia, gene expression profile, cyclophosphamide, drug resistance resistance. These results enable improved understanding of the mechanisms underlying drug resistance in CML cells.

\section{Introduction}

Chronic myelogenous leukemia (CML) is a type of myeloproliferative neoplasm predominantly characterized by uncontrolled growth of myeloid cells in the bone marrow and blood. It has an incidence of 1-2 cases per 100,000 adults, and accounts for $\sim 15 \%$ of all adult leukemia $(1,2)$. CML is composed of three distinct disease phases: An initial chronic phase (CP), an intermediate accelerated phase, and a terminal blast phase (BP) (3). In total, $\sim 90 \%$ of patients are diagnosed during the CP. The transition from CP to BP may occur as quickly as 3 years. The median survival rate is 3-6 months for CML patients in the BP without any treatment (4). The pathogenesis of CML has been well-described since 1960 (5). It is caused by a chromosomal translocation known as the Philadelphia chromosome $(\mathrm{Ph})$. This genetic translocation occurs between the Abelson murine leukemia $(A B L)$ gene located in chromosome 9 and the breakpoint cluster region $(B C R)$ gene in chromosome 22, resulting in a fusion gene called $B C R-A B L$ (6). The fused gene expresses an oncoprotein with a constitutively active tyrosine kinase that promotes cell growth and replication via regulating downstream pathways, including RAS, rapidly accelerated fibrosarcoma kinases, c-Jun N-terminal kinases, v-myc avian myelocytomatosis viral oncogene homolog and signal transducer and activator of transcription (STAT), resulting in the development of disease (7-11). Additional complex translocations have been reported in 5-8\% patients with CML, excluding chromosomes 9 and 22 . A t $(3 ; 9 ; 22) 3$-way translocation has been observed in patients with CML, who tend to have an aggressive stage and a poor outcome (12).

Patients with CML have multiple treatment options, including commercially available tyrosine kinase inhibitors (TKIs), omacetaxine, which inhibits protein synthesis, and several conventional anti-cancer agents (13). Allogeneic stem cell transplantation is a potential final option prior to CML progression, despite a high risk of mortality. However, its implementation depends on the status of patients and the identification of an appropriate stem cell donor (14). These therapeutic methods have improved the 10 -year overall survival rate to $80-90 \%(1,15)$. The majority of patients respond to first-line drug therapy; however, treatment failure 
still occurs in certain cases due to resistance and intolerance (16). Therefore, drug resistance is a current challenge faced by scientists and patients. It is critical to continue the identification of novel drug targets for patients with CML. Understanding the underlying mechanisms of drug resistance is also an emergent issue for researchers.

In the present study, the critical genes responsible for resistance of a conventional drug named cyclophosphamide were identified in patients with CML. Cyclophosphamide remains one of the most commonly used chemotherapy drug, 50 years subsequent to its synthesis. It is used as a single agent or in combination with other agents for multiple diseases, including solid tumors, hematologic malignancies, autoimmune disorders, stem-cell mobilization, and immunosuppression for blood and marrow transplantation (17). In spite of its wide therapeutic application, little is known about the mechanisms underlying the acquired resistance that is frequently observed in patients. In the present study, an Affymetrix microchip was used to identify differentially expressed genes between sensitive and resistant cells in response to treatment with cyclophosphamide. Analysis of KEGG pathways and protein-protein interactions was subsequently performed to reveal the potential factors mediating cyclophosphamide resistance in CML.

\section{Materials and methods}

Data collection. The Gene Expression Omnibus (GEO) database (www.ncbi.nlm.nih.gov/geo) was searched and microarray expression data (GSE7114) was obtained for two groups of chronic myelogenous leukemia cell lines that are either sensitive or resistant to cyclophosphamide treatment. The parental sensitive cell line was KBM-7/B5 and its 4-hydroperoxycyclophosphamide (4-HC)-resistant subline was B5-180. Each group had 5 biological replicates. Unprocessed data sets (.cel files) were collected for further analysis. The Affymetrix Human Genome U95 Version 2 Array was used in the experiments (Affymetrix; Thermo Fisher Scientific, Inc., Waltham, MA, USA). The probe annotation files were downloaded accordingly for further research.

Data processing and filtering. Several algorithms have been developed to quantify the microarray signal. GCRMA (version 2.36.0) (18) was used in the present study. The normalization process consisted of three steps: Model-based background correction, quantile normalization and summarizing.

In order to filter out uninformative data including control probe sets and other internal controls, as well as removing genes which were expressed uniformly close to background detection levels, the nsFilter function of the genefilter package (19) in R language was used. However, this filter does not remove probe sets without Entrez Gene identifiers or that have identical Entrez Gene identifiers.

Differentially-expressed gene (DEG) analysis. Statistical comparisons were performed between the sensitive and the resistant groups in response to cyclophosphamide treatment. Limma (20) in R language was applied to identify the differential expression of the comparison. For probes with identical Entrez Gene identifiers, only those probes that occupied the largest variance were kept for further DEG analysis. Only those genes with a $\log _{2}$ (fold change) $>1$ and adjusted P-value $<0.01$ were recognized as significantly differentially expressed between the two sample groups. The adjusted P-value was obtained through applying Benjamini and Hochberg's $(\mathrm{BH})$ false discovery rate correction on the original $\mathrm{P}$-value, and the fold change threshold was selected based on the focus of the present study on significantly DEGs.

Hierarchical clustering. Hierarchical clustering (21) was performed to classify the analyzed samples based on the gene expression profiles. Hierarchical clustering was performed using DEGs to observe the global gene expression patterns. The DEGs, which were classified in specific biological processes (GO terms) and KEGG pathway analysis, were further extracted and the expression pattern of those DEGs was characterized. The resultant heatmaps for the DEGs were classified as targeted biological processes or KEGG pathways using $\mathrm{R}$ package.

GO and KEGG pathway analysis. The R packages GO.db (22), KEGG.db (23) and KEGGREST (24) were utilized to detect Gene Ontology categories and KEGG pathways with significant overrepresentation in DEGs compared with the whole genome. The significantly enriched biological processes were identified as $\mathrm{P}<0.01$. For KEGG pathway analysis, the $\mathrm{P}$-value was set to $<0.05$.

Construction of biological network. Protein-protein interaction (PPI) databases were downloaded from the HPRD (25), BIOGRID (26), and PIP (27) databases. The pair interactions, which were included in any of the three databases, were selected to be included in the curated PPI database of the present study. As a result, 561,405 pair interactions were collected in this database. Cytoscape (28) was utilized to construct a PPI network. Interacting gene pairs existing in this curated PPI database were imported as a stored network. Following functional enrichment analysis, DEGs specified in dramatically altered biological processes (GO terms) and KEGG pathways were mapped to the corresponding networks to analyze the interactions.

Reverse transcription-quantitative polymerase chain reaction $(R T-q P C R)$. A total of 5 genes were selected for RT-qPCR, in order to validate the microarray data. The human CML cell lines K-562 and KU812, cultured following ATCC's methods, were treated with 0,10 or $100 \mu \mathrm{g} / \mathrm{ml} 4$-HC for $30 \mathrm{~min}$. K-562 was cultured in ATCC-formulated Iscove's modified Dulbecco's medium (catalog no. 30-2005) supplemented with $10 \%$ fetal bovine serum (FBS) and KU812 was cultured in ATCC-formulated RPMI-1640 medium (ATCC 30-2001) supplemented with $10 \%$ FBS. Total RNA was isolated using the RNeasy Mini kit (Qiagen, Inc., Valencia, CA, USA) according to the manufacturer's protocol. Following the manufacturer's protocol, reverse transcription and qPCR were performed using the High-Capacity cDNA Reverse Transcription kit and SYBR Green PCR kit, respectively (both Invitrogen; Thermo Fisher Scientific, Inc.). Results were quantified using the delta $\mathrm{Cq}$ method (29). The thermocycling conditions were as follows: $50^{\circ} \mathrm{C}$ for $2 \mathrm{~min}, 1$ cycle; $95^{\circ} \mathrm{C}$ for $10 \mathrm{~min}, 1$ cycle; $95^{\circ} \mathrm{C}$ $15 \mathrm{sec}->60^{\circ} \mathrm{C} 30 \mathrm{sec}->72^{\circ} \mathrm{C} 30 \mathrm{sec}, 40$ cycles; and finally $72^{\circ} \mathrm{C}$ 10 min, 1 cycle. 
The primer sequences used for PCR amplification were as follows: Spleen associated tyrosine kinase $(S Y K)$ forward, 5'-GTGTCATTCAATCCGTATGAGCC-3' and reverse, 5'-TTTCGGTCCAGGTAAACCTCC-3'; aldehyde dehydrogenase 2 family (mitochondrial; $A L D H 2$ ) forward, 5'-ATG GCAAGCCCTATGTCATCT-3', and reverse, 5'-CCGTGG TACTTATCAGCCCA-3'; midkine (neurite growth-promoting factor 2; $M D K$ ) forward, 5'-CGCGGTCGCCAAAAAGAA AG-3' and reverse, 5'-TACTTGCAGTCGGCTCCAAAC-3'; signal transducer and activator of transcription 5a (STAT5A) forward, 5'-GCAGAGTCCGTGACAGAGG-3', and reverse, 5'-CCACAGGTAGGGACAGAGTCT-3'; S100 calcium binding protein A4 (S100A4) forward, 5'-GATGAGCAACTT GGACAGCAA-3', and reverse, 5'-CTGGGCTGCTTATCT GGGAAG-3'; human 18s forward primer: 5'-GTAACCCGT TGAACCCCATT-3' and reverse, 5'-CCATCCAATCGGTAG TAGCG-3'. Gene expression was normalized to $18 \mathrm{~s}$.

RNA interference. To investigate the effect of the STAT5A and S100A4 genes on sensitivity to 4-HC in K-562 cells, expression of the STAT5A and S100A4 genes was inhibited by small interfering RNA (siRNA). K-562 cells were seeded into 6-well plates at $1 \times 10^{5}$ cells per well and cultured in medium without antibiotics following ATCC methods. Cells were transfected following $24 \mathrm{~h}$ culture with siRNAs at a final concentration of $40 \mathrm{nM}$ using Lipofectamine RNAiMAX (Ambion; Thermo Fisher Scientific, Inc.). Commercially available Silencer Select Pre-designed siRNAs for STAT5A, S100A4 and a negative control (Ambion; Thermo Fisher Scientific, Inc.) were used. The sequences of siRNAs for STAT5A and S100A4 were 5'-ATGGTTTCAGGTTCCACAG-3' and 5'-TGAGCT TGAACTTGTCACC-3', respectively. The sequence for the Control-siRNA was 5'-UAAGGCUAUGAAGAGAUAC-3'. Cells were subsequently collected for $\mathrm{mRNA}$ isolation following transfection for $36 \mathrm{~h}$.

In vitro 4-HC treatment assay. To investigate the inhibition of STAT5A and S100A4 on sensitivity to 4-HC, K-562 cells $\left(5 \times 10^{4}\right.$ cells $\left./ \mathrm{ml}\right)$ from each experimental group (siControl, siSTAT5A, siS100A4) were treated with 0,10 or $15 \mu \mathrm{g} / \mathrm{ml}$ 4-HC and incubated for $30 \mathrm{~min}$ at $37^{\circ} \mathrm{C}$. Following 4-HC treatment, cells were washed twice with chilled culture medium (RPMI; ATCC 30-2001) with 10\% fetal bovine serum (FBS; ATCC 30-2020), then plated. K-562 cells were plated in methylcellulose containing 25\% FBS or in liquid culture in RPMI containing 10\% FBS. Colonies were counted on day 7 of methylcellulose cultures with an inverted microscope (IX53; Olympus Corporation, Tokyo, Japan) under x10 magnification. A total of three fields were assessed from each group using Olympus CellSens ${ }^{\mathrm{TM}}$ Microscope Imaging Software (Olympus Corporation) to count the number of cells. The total number of viable cells in liquid cultures was determined twice within a 7-day period. Viability was determined using trypan blue exclusion criteria.

Statistical analysis. Data are presented as the mean \pm standard deviation. The statistical significance of the differences between experimental groups was calculated using GraphPad Instat 3 (GraphPad Software, Inc., La Jolla, CA, USA). A paired t-test was used for difference analysis between two
Table I. Statistical distribution of DEGs.

\begin{tabular}{lcc}
\hline DEGs & Probe & Gene \\
\hline Total DEGs & 5885 & 4824 \\
Significantly DEGs ${ }^{\mathrm{a}}$ & $300(167 / 133)^{\mathrm{b}}$ & $258(139 / 119)^{\mathrm{b}}$ \\
\hline${ }^{\mathrm{a} \log _{2} \text { (Fold Change) }>1 \text { and adjusted P-value }<0.01 .}{ }^{\mathrm{b}}$ (upregu- \\
lated/downregulated). DEG, differentially-expressed gene.
\end{tabular}

groups and one-way analysis of variance was used for the comparison of three or more groups. $\mathrm{P}<0.05$ was considered to indicate a statistically significant difference.

\section{Results}

DEG analysis. The publicly available microarray dataset GSE7114 was obtained from the GEO database. Comparative analysis was performed between sensitive groups and resistant groups to identify genes with significantly differential expression. At $\log _{2}$ (fold change) $>1$ and adjusted P-value $<0.01$, a total of 258 DEGs were identified, among which 139 DEGs were upregulated and 119 DEGs were downregulated (Table I). The top 50 upregulated and downregulated DEGs were listed in Tables II and III, respectively. Several potential genes connected with cyclophosphamide resistance were identified from these DEGs, including aldehyde dehydrogenase 1 family member 1 (ALDH1A1), ALDH2, aldo-keto reductase family 1 member B (AKR1B1), MDK, S100A4 and TIMP metallopeptidase inhibitor 1 (TIMP1). All of these were previously known to be associated with drug-resistance, and were primarily functionally linked to drug metabolism, cell proliferation and the anti-apoptotic process.

Construction of the biological network. A heatmap of hierarchical clustering of all DEGs was generated to visualize differential gene expression status between the sensitive groups and resistant groups (Fig. 1A). In order to further verify the biological networks, all significantly regulated PPIs identified from the HPRD, BIOGRID, and PIP databases were utilized to construct a biological network using cytoscape software. Several sub-networks were indicated (Fig. 1B). The majority of the proteins were involved in one or more sub-networks. The three central genes that constituted the hubs of the network were STAT5A, FYN proto-oncogene, Src family tyrosine kinase $(F Y N)$ and $S Y K$, suggesting that these may be involved in cyclophosphamide resistance in patients with CML. As the network of all DEGs was too complex to successfully elucidate the function of sub-networks, detailed analysis was required.

Biological processes analysis. The differentially expressed genes determined by microarray analysis were subjected to GO and KEGG pathway analysis. The data were generated based on GO terms with P-values $<0.01$ to identify biological processes. A total of 487 biological processes that were overrepresented by DEGs were identified (Table IV). Table V lists the top six significantly enriched biological processes, including regulation of biological quality, immune system process, response 
Table II. Top 50 upregulated genes $\left[\log _{2}\right.$ (fold change) $>1$ and adjusted P-value $<0.01]$.

\begin{tabular}{|c|c|c|c|}
\hline $\begin{array}{l}\text { Gene } \\
\text { symbol }\end{array}$ & $\begin{array}{c}\log _{2} \\
\text { (fold change) }\end{array}$ & $\begin{array}{c}\text { Adj. } \\
\text { P-value }\end{array}$ & P-value \\
\hline$A L D H 1 A 1$ & 175.23 & $2.12 \times 10^{-11}$ & $3.38 \times 10^{-15}$ \\
\hline LGALS1 & 25.05 & $2.14 \times 10^{-07}$ & $6.13 \times 10^{-10}$ \\
\hline $\mathrm{CTSH}$ & 24.08 & $7.97 \times 10^{-08}$ & $1.65 \times 10^{-10}$ \\
\hline$A L D H 2$ & 17.36 & $1.20 \times 10^{-10}$ & $3.81 \times 10^{-14}$ \\
\hline$M A R C K S$ & 11.73 & $8.73 \times 10^{-10}$ & $4.17 \times 10^{-13}$ \\
\hline$R A P G E F 2$ & 11.72 & $4.00 \times 10^{-06}$ & $3.03 \times 10^{-08}$ \\
\hline$M D K$ & 11.08 & $1.42 \times 10^{-05}$ & $1.89 \times 10^{-07}$ \\
\hline IDI & 10.68 & $4.72 \times 10^{-07}$ & $2.18 \times 10^{-09}$ \\
\hline FSCN1 & 8.30 & $6.66 \times 10^{-06}$ & $6.66 \times 10^{-08}$ \\
\hline$P M P 22$ & 8.23 & $1.80 \times 10^{-07}$ & $4.29 \times 10^{-10}$ \\
\hline SIGLEC6 & 8.19 & $5.37 \times 10^{-07}$ & $2.65 \times 10^{-09}$ \\
\hline$G L R B$ & 8.06 & $2.62 \times 10^{-07}$ & $9.35 \times 10^{-10}$ \\
\hline CTSL & 7.66 & $1.27 \times 10^{-09}$ & $8.09 \times 10^{-13}$ \\
\hline$A N G P T 1$ & 7.31 & $2.81 \times 10^{-09}$ & $3.13 \times 10^{-12}$ \\
\hline$I T G A 2 B$ & 6.93 & $1.07 \times 10^{-05}$ & $1.32 \times 10^{-07}$ \\
\hline$R A B 31$ & 6.71 & $7.81 \times 10^{-09}$ & $9.95 \times 10^{-12}$ \\
\hline$R U N D C 3 B$ & 6.69 & $2.81 \times 10^{-09}$ & $3.10 \times 10^{-12}$ \\
\hline DUSP6 & 6.30 & $1.07 \times 10^{-05}$ & $1.31 \times 10^{-07}$ \\
\hline ICAM2 & 6.04 & $4.62 \times 10^{-06}$ & $3.87 \times 10^{-08}$ \\
\hline$K L F 1$ & 5.91 & $4.66 \times 10^{-06}$ & $4.01 \times 10^{-08}$ \\
\hline IFI44 & 5.77 & $2.52 \times 10^{-08}$ & $4.01 \times 10^{-11}$ \\
\hline S100A4 & 5.50 & $1.16 \times 10^{-05}$ & $1.48 \times 10^{-07}$ \\
\hline ACSM3 & 5.47 & $7.74 \times 10^{-03}$ & $8.22 \times 10^{-04}$ \\
\hline PAX6 & 5.42 & $5.92 \times 10^{-06}$ & $5.42 \times 10^{-08}$ \\
\hline$F Y N$ & 5.40 & $4.00 \times 10^{-06}$ & $2.95 \times 10^{-08}$ \\
\hline$C P V L$ & 5.17 & $1.04 \times 10^{-05}$ & $1.23 \times 10^{-07}$ \\
\hline$R R A S 2$ & 5.08 & $1.64 \times 10^{-08}$ & $2.35 \times 10^{-11}$ \\
\hline$D L C 1$ & 5.04 & $4.62 \times 10^{-06}$ & $3.90 \times 10^{-08}$ \\
\hline YES1 & 5.02 & $4.81 \times 10^{-05}$ & $9.20 \times 10^{-07}$ \\
\hline$L C P 2$ & 4.89 & $7.20 \times 10^{-07}$ & $4.01 \times 10^{-09}$ \\
\hline$L A T$ & 4.87 & $2.06 \times 10^{-06}$ & $1.35 \times 10^{08}$ \\
\hline TPST2 & 4.84 & $2.59 \times 10^{-07}$ & $8.25 \times 10^{-10}$ \\
\hline STEAP1 & 4.53 & $6.42 \times 10^{-06}$ & $6.03 \times 10^{-08}$ \\
\hline$K A Z N$ & 4.26 & $7.52 \times 10^{-07}$ & $4.53 \times 10^{-09}$ \\
\hline$C D 48$ & 4.07 & $1.27 \times 10^{-04}$ & $3.48 \times 10^{-06}$ \\
\hline$I L 2 R A$ & 4.04 & $2.84 \times 10^{-03}$ & $2.20 \times 10^{-04}$ \\
\hline COL $2 A 1$ & 4.03 & $3.37 \times 10^{-04}$ & $1.28 \times 10^{-05}$ \\
\hline$T A L 1$ & 3.98 & $2.82 \times 10^{-04}$ & $1.03 \times 10^{-05}$ \\
\hline PRAME & 3.96 & $8.47 \times 10^{-05}$ & $2.04 \times 10^{-06}$ \\
\hline TNFSF 10 & 3.91 & $2.71 \times 10^{-03}$ & $2.06 \times 10^{-04}$ \\
\hline$A S S 1$ & 3.80 & $1.28 \times 10^{-04}$ & $3.52 \times 10^{-06}$ \\
\hline GUSBP11 & 3.72 & $8.09 \times 10^{-03}$ & $8.71 \times 10^{-04}$ \\
\hline TIMP1 & 3.61 & $3.34 \times 10^{-05}$ & $5.64 \times 10^{-07}$ \\
\hline$Z H X 2$ & 3.60 & $6.98 \times 10^{-05}$ & $1.60 \times 10^{-06}$ \\
\hline STAR & 3.59 & $4.52 \times 10^{-04}$ & $1.92 \times 10^{-05}$ \\
\hline PHLDA2 & 3.52 & $9.21 \times 10^{-06}$ & $1.03 \times 10^{-07}$ \\
\hline$T S C 22 D 1$ & 3.46 & $4.05 \times 10^{-07}$ & $1.68 \times 10^{-09}$ \\
\hline ID3 & 3.43 & $4.57 \times 10^{-05}$ & $8.37 \times 10^{-07}$ \\
\hline$A K R 1 B 1$ & 3.32 & $3.67 \times 10^{-04}$ & $1.47 \times 10^{-05}$ \\
\hline PICALM & 3.30 & $1.22 \times 10^{-04}$ & $3.17 \times 10^{-06}$ \\
\hline
\end{tabular}

Table III. Top 50 downregulated genes $\left[\log _{2}\right.$ (fold change) $<-1$ and adjusted P-value <0.01].

\begin{tabular}{|c|c|c|c|}
\hline $\begin{array}{l}\text { Gene } \\
\text { symbol }\end{array}$ & $\begin{array}{c}\log _{2} \\
\text { (fold change) }\end{array}$ & $\begin{array}{c}\text { Adj. } \\
\text { P-value }\end{array}$ & P-value \\
\hline PRG2 & 152.01 & $3.48 \times 10^{-07}$ & $1.33 \times 10^{-09}$ \\
\hline$C A 2$ & 40.15 & $2.14 \times 10^{-07}$ & $5.50 \times 10^{-10}$ \\
\hline$M S 4 A 3$ & 33.15 & $6.47 \times 10^{-07}$ & $3.30 \times 10^{-09}$ \\
\hline PRTN3 & 28.69 & $2.23 \times 10^{-07}$ & $6.75 \times 10^{-10}$ \\
\hline CST7 & 28.36 & $8.19 \times 10^{-08}$ & $1.83 \times 10^{-10}$ \\
\hline IL32 & 21.11 & $1.83 \times 10^{-05}$ & $2.59 \times 10^{-07}$ \\
\hline RNASE2 & 19.05 & $1.51 \times 10^{-05}$ & $2.07 \times 10^{-07}$ \\
\hline COX7A2 & 17.67 & $3.53 \times 10^{-07}$ & $1.41 \times 10^{-09}$ \\
\hline CEBPE & 12.49 & $5.32 \times 10^{-08}$ & $9.71 \times 10^{-11}$ \\
\hline EPB41L2 & 8.47 & $4.17 \times 10^{-07}$ & $1.86 \times 10^{-09}$ \\
\hline$A Z U 1$ & 8.13 & $1.84 \times 10^{-04}$ & $5.76 \times 10^{-06}$ \\
\hline GBE1 & 7.72 & $1.72 \times 10^{-05}$ & $2.42 \times 10^{-07}$ \\
\hline CCR 8 & 7.45 & $1.85 \times 10^{-05}$ & $2.66 \times 10^{-07}$ \\
\hline$C F D$ & 6.59 & $1.37 \times 10^{-04}$ & $3.87 \times 10^{-06}$ \\
\hline S100P & 6.49 & $2.31 \times 10^{-03}$ & $1.64 \times 10^{-04}$ \\
\hline$A L O X 5 A P$ & 5.59 & $3.28 \times 10^{-03}$ & $2.67 \times 10^{-04}$ \\
\hline$R R A G A$ & 5.48 & $7.09 \times 10^{-07}$ & $3.72 \times 10^{-09}$ \\
\hline SRGN & 5.36 & $1.25 \times 10^{-04}$ & $3.34 \times 10^{-06}$ \\
\hline NDRG1 & 5.06 & $1.28 \times 10^{-03}$ & $7.55 \times 10^{-05}$ \\
\hline BNIP3 & 4.89 & $3.95 \times 10^{-03}$ & $3.44 \times 10^{-04}$ \\
\hline TEX30 & 4.81 & $4.00 \times 10^{-06}$ & $3.01 \times 10^{-08}$ \\
\hline CORO2A & 4.76 & $7.20 \times 10^{-07}$ & $4.13 \times 10^{-09}$ \\
\hline$B P I$ & 4.75 & $4.71 \times 10^{-04}$ & $2.03 \times 10^{-05}$ \\
\hline KBTBD 11 & 4.35 & $3.92 \times 10^{-05}$ & $6.75 \times 10^{-07}$ \\
\hline$E B P$ & 4.23 & $1.32 \times 10^{-05}$ & $1.74 \times 10^{-07}$ \\
\hline SERINC5 & 4.21 & $4.01 \times 10^{-06}$ & $3.13 \times 10^{-08}$ \\
\hline$P 4 H B$ & 4.12 & $4.83 \times 10^{-07}$ & $2.31 \times 10^{-09}$ \\
\hline$I T M 2 A$ & 4.12 & $6.50 \times 10^{-05}$ & $1.45 \times 10^{-06}$ \\
\hline$R X R A$ & 4.07 & $4.43 \times 10^{-04}$ & $1.87 \times 10^{-05}$ \\
\hline ICAM3 & 3.97 & $2.36 \times 10^{-06}$ & $1.58 \times 10^{-08}$ \\
\hline ATF5 & 3.75 & $2.53 \times 10^{-04}$ & $8.87 \times 10^{-06}$ \\
\hline$W W P 1$ & 3.60 & $1.26 \times 10^{-04}$ & $3.40 \times 10^{-06}$ \\
\hline$A R F G E F 1$ & 3.52 & $3.00 \times 10^{-05}$ & $4.91 \times 10^{-07}$ \\
\hline PRDX2 & 3.42 & $7.52 \times 10^{-07}$ & $4.55 \times 10^{-09}$ \\
\hline$S Y K$ & 3.42 & $2.38 \times 10^{-05}$ & $3.67 \times 10^{-07}$ \\
\hline$M X 2$ & 3.35 & $2.03 \times 10^{-05}$ & $2.97 \times 10^{-07}$ \\
\hline$L M O 2$ & 3.25 & $2.78 \times 10^{-04}$ & $1.00 \times 10^{-05}$ \\
\hline CCNE2 & 3.23 & $3.00 \times 10^{-03}$ & $2.39 \times 10^{-04}$ \\
\hline$D G A T 1$ & 3.22 & $4.64 \times 10^{-05}$ & $8.77 \times 10^{-07}$ \\
\hline EFR3A & 3.13 & $7.20 \times 10^{-07}$ & $4.11 \times 10^{-09}$ \\
\hline PTDSS1 & 3.06 & $1.29 \times 10^{-05}$ & $1.69 \times 10^{-07}$ \\
\hline$H L A-A$ & 3.00 & $1.92 \times 10^{-05}$ & $2.78 \times 10^{-07}$ \\
\hline$C Y B 5 A$ & 2.95 & $3.29 \times 10^{-04}$ & $1.24 \times 10^{-05}$ \\
\hline TNFAIPS & 2.92 & $2.62 \times 10^{-07}$ & $9.34 \times 10^{-10}$ \\
\hline$P E X 2$ & 2.92 & $2.45 \times 10^{-04}$ & $8.42 \times 10^{-06}$ \\
\hline$M A D 1 L 1$ & 2.88 & $1.72 \times 10^{-05}$ & $2.38 \times 10^{-07}$ \\
\hline SAT1 & 2.87 & $1.25 \times 10^{-03}$ & $7.31 \times 10^{-05}$ \\
\hline$N C A L D$ & 2.83 & $2.61 \times 10^{-03}$ & $1.95 \times 10^{-04}$ \\
\hline$C E B P D$ & 2.82 & $6.66 \times 10^{-06}$ & $6.54 \times 10^{-08}$ \\
\hline$I K Z F 1$ & 2.80 & $2.23 \times 10^{-03}$ & $1.56 \times 10^{-04}$ \\
\hline
\end{tabular}


A

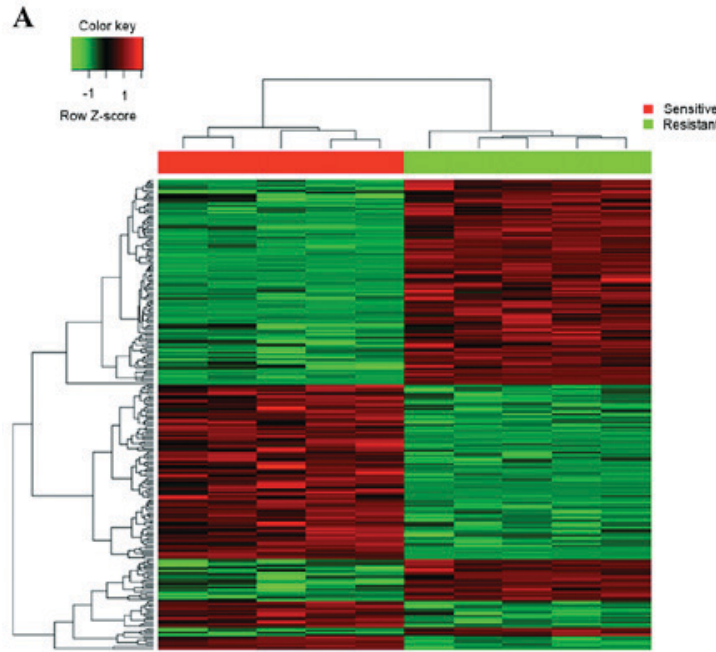

B

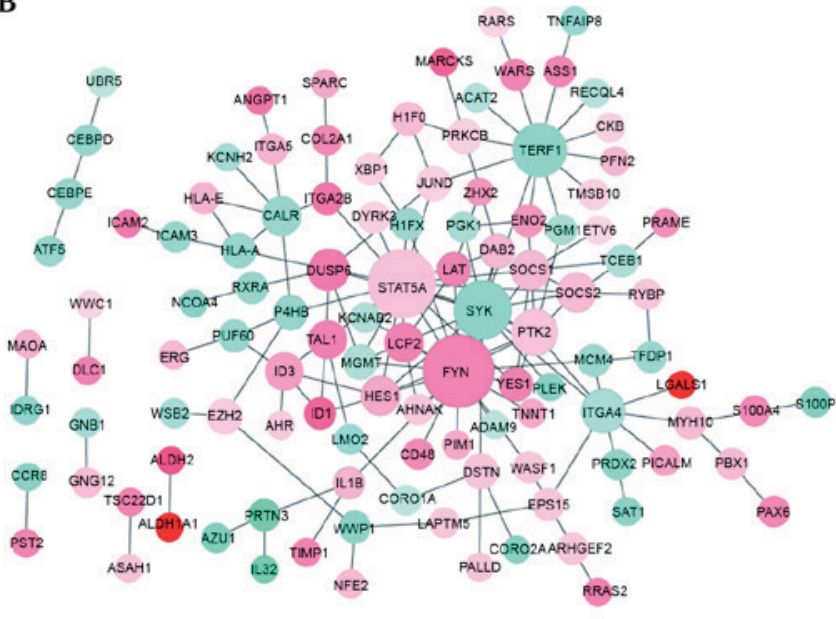

Figure 1. Heat map of all DEGs and corresponding biological networks. (A) Heat map of hierarchical clustering of all DEGs (5 resistant samples and 5 sensitive samples). Red indicates high relative expression and green indicates low relative expression. (B) A biological network was constructed according to the interactions between all DEGs. Pink indicates upregulated expression and green indicates down-regulated expression. The darker the color, the greater the difference. DEG, differentially-expressed gene.

Table IV. Obtained GO biological processes and KEGG pathways.

\begin{tabular}{lcc}
\hline Analysis & P-value & No. \\
\hline GO biological process & $<0.01$ & 487 \\
KEGG pathway & $<0.05$ & 17 \\
\hline
\end{tabular}

GO, Gene Ontology; KEGG, Kyoto Encyclopedia of Genes and Genomes.

Table V. Top six significantly altered GO biological processes.

\begin{tabular}{llrl}
\hline GO-BP-ID & P-value & Count & \multicolumn{1}{c}{ Term } \\
\hline GO:0065008 & $4.09 \times 10^{-15}$ & 98 & $\begin{array}{l}\text { Regulation of biological } \\
\text { quality }\end{array}$ \\
GO:0002376 & $4.82 \times 10^{-15}$ & 82 & $\begin{array}{l}\text { Immune system process } \\
\text { GO:0042221 }\end{array}$ \\
$1.70 \times 10^{-12}$ & 98 & Response to chemical \\
GO:0006915 & $2.45 \times 10^{-09}$ & 59 & Apoptotic process \\
GO:0023052 & $1.08 \times 10^{-08}$ & 123 & Signaling pathway \\
GO:0008283 & $1.32 \times 10^{-08}$ & 58 & Cell proliferation \\
\hline
\end{tabular}

GO, Gene Ontology.

to chemicals, apoptotic process, signaling pathways and cell proliferation. Heatmaps and biological networks of the six significant biological processes were generated: Biological quality (Fig. 2A), immune system process (Fig. 2B), response to chemicals (Fig. 2C), signaling pathways (Fig. 2D), apoptotic process (Fig. 2E) and cell proliferation (Fig. 2F). This network analysis identified a number of potential genes that may be associated with cyclophosphamide resistance. Therefore, GO analysis provided a valuable mechanistic insight into cyclophosphamide resistance in CML cells.

KEGG pathway enrichment of DEGs. For KEGG pathway enrichment analysis, a count $>4$ and a P-value $<0.05$ were set as the threshold, and 17 KEGG pathways were identified (Table IV). The top significant KEGG pathways were hematopoietic cell lineage, natural killer cell mediated cytotoxicity, arginine and proline metabolism, lysosome, phagosome, osteoclast differentiation and Fc epsilon RI signaling pathway (Table VI). Heatmaps and biological networks of KEGG pathways were generated, including natural killer cell mediated cytotoxicity (Fig. 3A), phagosome (Fig. 3B), osteoclast differentiation (Fig. 3C) and the Fc epsilon RI signaling pathway (Fig. 3D). The major genes identified from these networks were those involved in the cell cycle, proliferation, signaling transduction, cell adhesion and the immune response.

Identification of central genes from signal networks. The analysis of networks for biological processes and KEGG pathways permitted the identification of genes involved in these processes and pathways, as well as associations between upstream and downstream signaling transduction. The results revealed that three genes (STAT5A, FYN and $S Y K$ ) were frequently present as hubs of corresponding networks, highlighting their functions as important markers for cyclophosphamide resistance.

Validation of microarray data. To verify the expression of the DEGs identified in microarray experiments, RT-qPCR was performed using two human CML cell lines (K-562 and KU812). The expression levels of 5 genes were tested ( $S Y K$, $A L D H 2, M D K, S T A T 5 A$ and S100A4), and mRNA expression levels of SYK were significantly decreased and mRNA expression levels of ALDH2, MDK, STAT5A and S100A4 were significantly increased following $4-\mathrm{HC}$ treatment in 


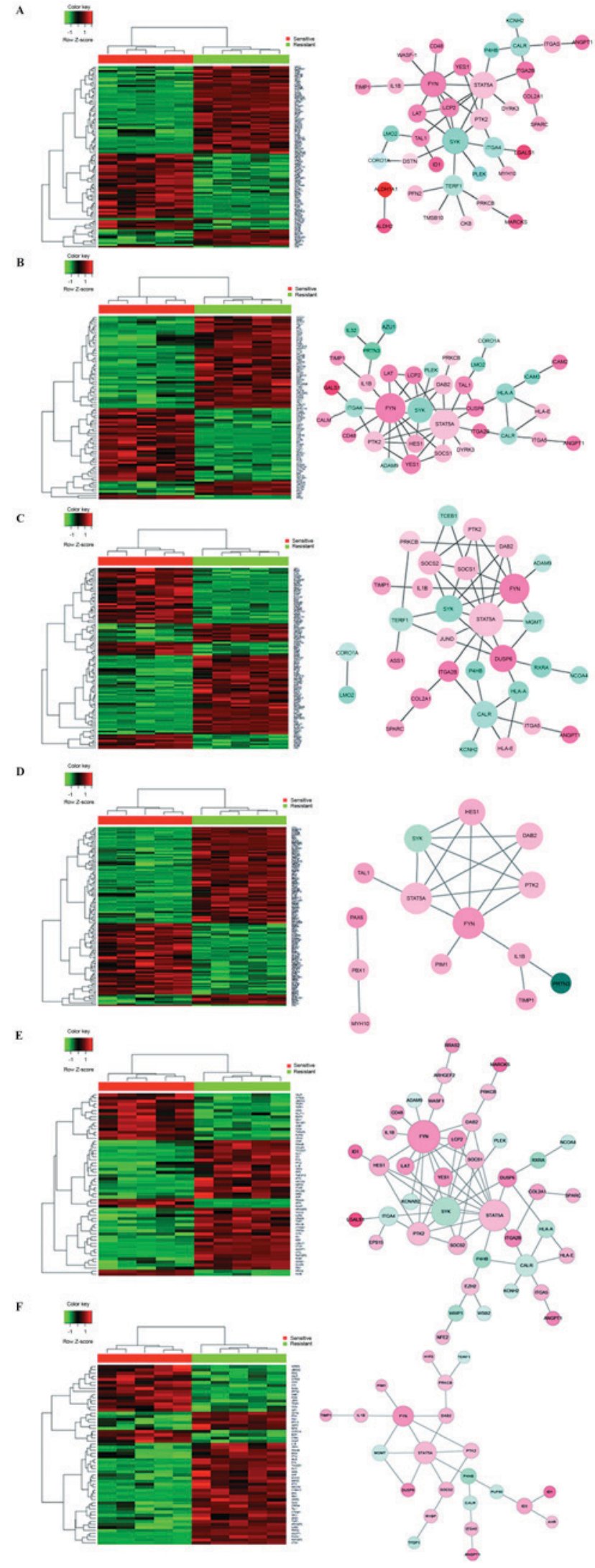

Figure 2. Heat maps and corresponding biological networks of the top six significantly altered biological processes: (A) regulation of biological quality (GO:0065008), (B) immune system process (GO:0002376), (C) response to chemicals (GO:0042221), (D) signaling pathways (GO:00,23052), (E) apoptotic process (GO:0006915) and (F) cell proliferation (GO:0008283). Red indicates high relative expression and green indicates low relative expression in the heat maps. For network analysis, pink indicates upregulated expression and green indicates down-regulated expression. The darker the color, the greater the difference. GO, gene ontology.
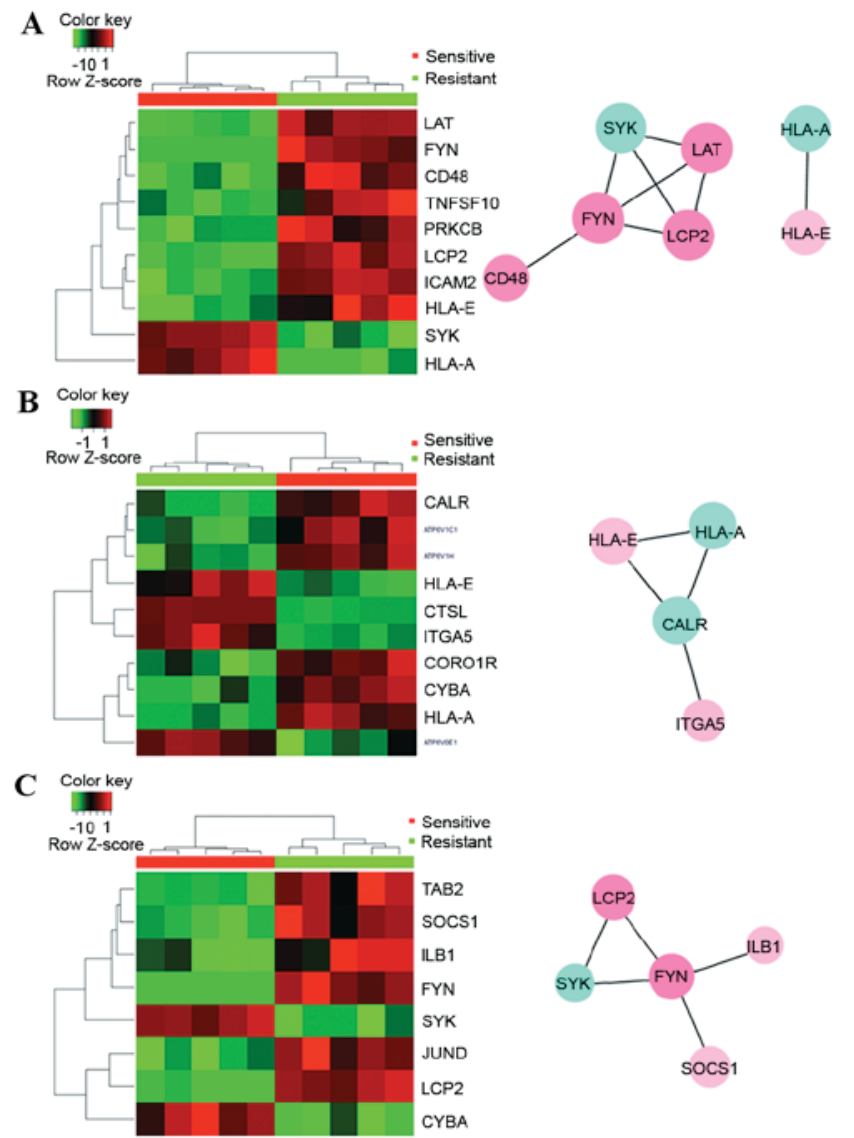

D
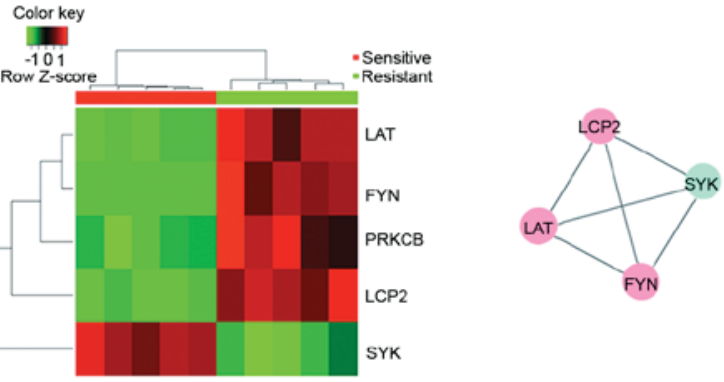

Figure 3. Heat maps and corresponding biological networks of several significantly altered Kyoto Encyclopedia of Genes and Genomes pathways: (A) natural killer cell mediated cytotoxicity, (B) phagosome, (C) osteoclast differentiation and (D) Fc epsilon RI signaling pathway, respectively. Red indicates high relative expression and green indicates low relative expression in the heat maps. For network analysis, pink indicates upregulated expression and green indicates down-regulated expression. The darker the color, the greater the difference. GO, gene ontology.

K-562 (Fig. 4A) and KU812 cells (Fig. 4B). These results were in agreement with and supported the microarray data.

Effect of STAT5A and S100A4 on sensitivity to 4-HC. To further determine whether high expression of resistance-associated genes conferred decreased sensitivity to $4-\mathrm{HC}$, the genes STAT5A and S100A4, which were highly expressed in K-562 cells following treatment with 4-HC, were used to assess their potentially drug resistant function in vitro. The mRNA expression levels of STAT5A and S100A4 were efficiently inhibited by interference with siRNA method, when compared with the control (Fig. 5A and B, respectively). Following transfection, cells were treated with 0,10 or $15 \mu \mathrm{g} / \mathrm{ml} 4-\mathrm{HC}$ and cultured in methylcellulose culture or liquid culture. Table VII 
Table VI. Significantly altered KEGG pathways.

\begin{tabular}{|c|c|c|c|}
\hline KEGG-ID & P-value & Count & Term \\
\hline 04640 & $6.78 \times 10^{-4}$ & 8 & Hematopoietic cell lineage \\
\hline 04650 & $8.02 \times 10^{-4}$ & 10 & $\begin{array}{l}\text { Natural killer cell mediated } \\
\text { Cytotoxicity }\end{array}$ \\
\hline 00330 & $1.14 \times 10^{-3}$ & 6 & $\begin{array}{l}\text { Arginine and proline } \\
\text { metabolism }\end{array}$ \\
\hline 04142 & $1.35 \times 10^{-3}$ & 9 & Lysosome \\
\hline 04145 & $1.97 \times 10^{-3}$ & 10 & Phagosome \\
\hline 04380 & $7.22 \times 10^{-3}$ & 8 & Osteoclast differentiation \\
\hline 04664 & $3.02 \times 10^{-2}$ & 5 & $\begin{array}{l}\text { Fc epsilon RI signaling } \\
\text { pathway }\end{array}$ \\
\hline
\end{tabular}

Table VII. Effect of inhibition of STAT5A and S100A4 genes on the sensitivity of K-562 cells to 4-HC.

\begin{tabular}{lccc}
\hline $\begin{array}{l}4-\mathrm{HC} \text { dose } \\
(\mu \mathrm{g} / \mathrm{ml})\end{array}$ & siControl & siSTAT5A & siS100A4 \\
\hline 10 & $116 \pm 12^{\mathrm{a}}$ & $65 \pm 5^{\mathrm{b}}$ & $56 \pm 4^{\mathrm{b}}$ \\
& $(1.15)$ & $(0.43)$ & $(0.38)$ \\
15 & $30 \pm 3$ & $8 \pm 2^{\mathrm{b}}$ & $5 \pm 1^{\mathrm{b}}$ \\
& $(0.297)$ & $(0.053)$ & $(0.034)$ \\
\hline
\end{tabular}

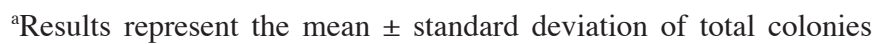
scored in five replicate methylcellulose culture plates, plated with $5 \times 10^{4}$ 4-HC-treated cells. Values in parentheses represent percentage recovery of colonies from 4-HC-treated cells in relation to the number of colonies obtained from the matched untreated cells. (The same number of untreated cells was cultured). ' ${ }^{\text {Significantly }}$ different compared with siControl group; $\mathrm{P}<0.005$. STAT5A, signal transducer and activator of transcription 5a; S100A4, S100 calcium binding protein A4; 4-HC, hydroperoxycyclophosphamide; si, small interfering RNA.

presents the results as the mean number of total colonies counted in 5 replicate experiments. K-562 cells expressing lower levels of the STAT5A gene were more sensitive to 4-HC when compared with the control. Similar results were observed for the S100A4 gene following transfection, which also increased the sensitivity of K-562 cells to 4-HC.

\section{Discussion}

Cyclophosphamide is an alkylating agent that exists as an inactive prodrug that requires enzymatic and chemical activation to release active phosphoramide mustard. Phosphoramide mustard alkylates DNA to produce interstrand and intrastrand DNA crosslinks, thus resulting in inhibition of DNA synthesis and cell death (30). Although the aldehyde dehydrogenase (ALDH) family has been reported to confer resistance to cyclophosphamide in multiple tumor cell lines (31), explaining cases with cyclophosphamide resistance remains difficult. In particular, patients with leukemia and lymphoma with low levels of ALDH isozymes are relatively resistant to cyclophosphamide (32). Therefore, it is urgent to improve understanding of the mechanisms of cyclophosphamide resistance, in order to use it in a more efficient way.

In the present study, the Affymetrix Human Genome U95 Version 2 microarray was used to analyze the global gene expression profile between sensitive and resistant CML cells in response to cyclophosphamide. A total of 258 DEGs demonstrated significantly differential regulation, of which 139 DEGs were upregulated and 119 DEGs were downregulated. A biological network was subsequently constructed according to PPIs obtained from the HPRD, BIOGRID, and PIP databases. GO and KEGG pathway enrichment analysis were also performed to reveal the altered biological events associated with cyclophosphamide resistance.

Among the DEGs, several potential genes that may be responsible for cyclophosphamide resistance were identified. For example, $A L D H 1 A 1$ was the top upregulated gene with a $\log _{2}$ fold change of 175.23 , and its expression has been the major determinant of cellular sensitivity to cyclophosphamide $(31,33)$. Another ALDH family member, $A L D H 2$, was also revealed to be upregulated in resistant CML cells, highlighting the critical function of the ALDH family as a predictive marker for drug resistance. The ALDH family mediates detoxification of cyclophosphamide by catalyzing the oxidation of its intermediate metabolite aldophosphamide to carboxyphosphamide, resulting in inactivation of cyclophosphamide $(17,32)$.

Another upregulated enzyme that may be involved in drug resistance was $A K R 1 B 1$, an aldose reductase. Overexpression of three reductases (carbonyl reductase 1, aldo-keto reductase family 1 member $\mathrm{A} 1$ and $A K R 1 B 1$ ) has been demonstrated to inactivate the anti-cancer drug daunorubicin, resulting in elevation of chemoresistance in tumor cells (34).

$M D K$ is another DEG that demonstrated upregulation in cyclophosphamide-resistant CML cells when compared with sensitive cells. It is a heparin-binding growth factor involved in cancer development, the promotion of cell growth and survival, angiogenesis and anti-apoptosis (35). Notably, over-expression of $M D K$ has been demonstrated to be associated with resistance to different chemotherapeutic agents, including fluorouracil, doxorubicin, cisplatin and adriamycin (36,37). Therefore, acquired upregulation of $M D K$ in CML cells may contribute to cyclophosphamide resistance.

S100A4 is a member of the S100 family of calcium-binding proteins and is involved in a number of cellular processes, including cell proliferation, differentiation, apoptosis, tumorigenesis and cancer metastasis (38). In addition, knockdown of S100A4 has been demonstrated to result in an increased sensitivity to gemcitabine in pancreatic cancer cells, suggesting that overexpression of S100A4 is associated with chemoresistance (39). Therefore, the upregulation of S100A4 observed in the present study may explain the resistance to cyclophosphamide in CML cells.

TIMPI is another gene with a potential involvement in drug resistance. High TIMPI expression levels are related with poor prognosis and poor response to chemotherapy in patients with breast cancer (40). Similar to this previous study, TIMPI was increased in cyclophosphamide-resistant CML cells in the present study, supporting the use of TIMP1 as a marker for drug sensitivity. 
$\mathbf{A}$

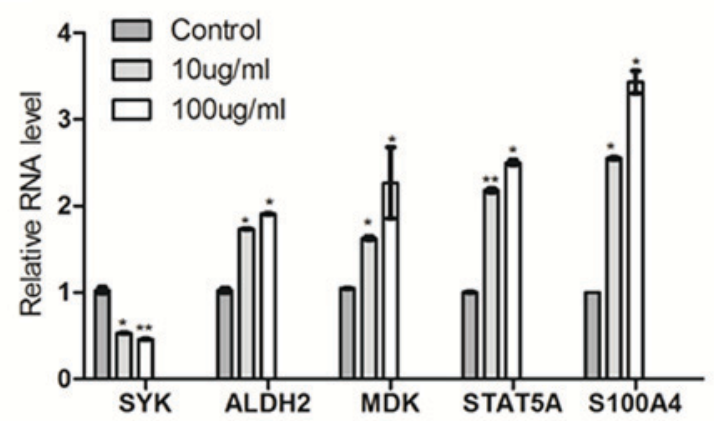

\section{B}

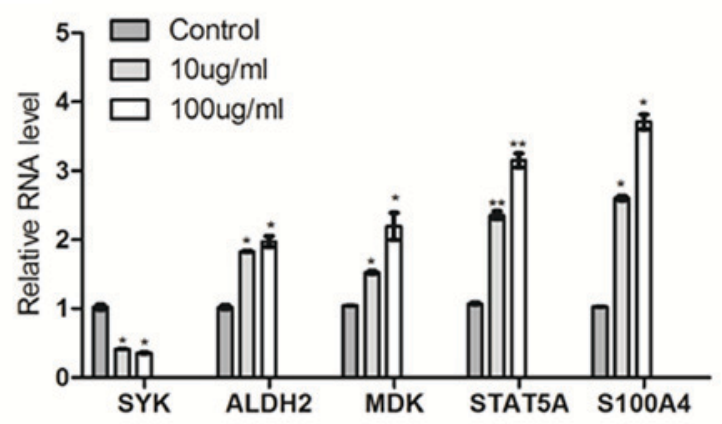

Figure 4. Confirmation of expression levels of five differentially expressed genes in (A) K-562 and (B) KU812 human chronic myelogenous leukemia cell lines. Cells were treated with 0,10 or $100 \mu \mathrm{g} / \mathrm{ml} 4$-hydroperoxycyclophohsphamide for $30 \mathrm{~min}$, and then collected for reverse transcription-quantitative polymerase chain reaction analysis. Error bars represent the mean \pm standard deviation of three independent experiments. ${ }^{*} \mathrm{P}<0.05$ and ${ }^{* *} \mathrm{P}<0.01$ vs. control. $S Y K$, spleen associated tyrosine kinase; $A L D H 2$, aldehyde dehydrogenase 2 family (mitochondrial); $M D K$, midkine (neurite growth-promoting factor 2); STAT5A, signal transducer and activator of transcription 5a; S100A4, S100 calcium binding protein $\mathrm{A} 4$.
A

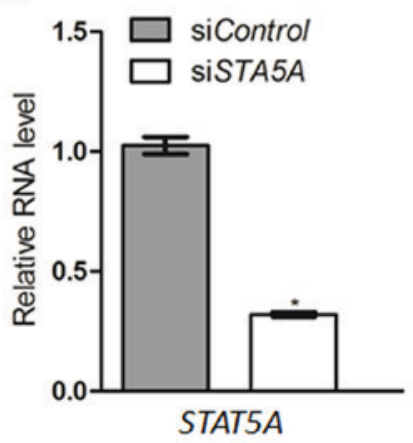

B

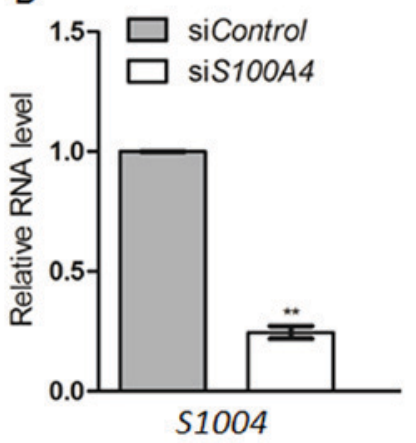

Figure 5. Inhibition of STAT5A and S100A4 expression in K-562 cells. Cells were transfected with siControl, siSTAT5A or siS100A4. mRNA expression levels of (A) STAT5A and (B) S100A4 were determined by reverse transcription-quantitative polymerase chain reaction following transfection. Error bars represent the mean \pm standard deviation of three independent experiments. ${ }^{*} \mathrm{P}<0.05$ and ${ }^{* *} \mathrm{P}<0.01$ vs. siControl. STAT5A, signal transducer and activator of transcription $5 \mathrm{~A} ; \mathrm{S} 100 \mathrm{~A} 4, \mathrm{~S} 100$ calcium binding protein A4; siControl, control siRNA; siSTAT5A, STAT5A-specific small interfering RNA; siS100A4, S100A4-specific small interfering RNA.

It is possible to use GO analysis to identify over-representation of biological processes, providing insights into mechanisms of chemoresistance in response to cyclophosphamide. Similar to the effects discussed previously, significant biological processes including the apoptotic process and cell proliferation were considered to be associated with drug resistance. It is reasonable to infer that the apoptosis processes induced in sensitive cells was inactive in resistant cells, thus promoting cell proliferation. The challenge is to dissect the key molecular mechanisms that drive these biological processes that occur in resistant cells. Response to chemical stimuli was another important biological event in the acquisition of drug resistance. The ALDH family was involved in the process of response to chemicals. The results of the present study suggested that cyclophosphamide-resistant CML cells may respond more effectively to external drug stimuli due to high levels of enzymes associated with clearance or inactivation of drugs, including the ALDH family.

KEGG enrichment analysis helps identify significant pathways that DEGs participate in, providing a comprehensive understanding of interactive genes. The pathway analysis performed in the present study revealed that 17 KEGG pathways appeared with a high frequency. The most significant pathways were involved in the immune response, including natural killer cell mediated cytotoxicity, lysosome, phagosome and Fc epsilon RI signaling pathway. These findings confirmed those of the GO analysis, confirming that the immune system process was one of the significantly enriched biological processes. The Fc epsilon RI complex forms a high affinity cell-surface receptor that interacts the Fc region of antigen-specific immunoglobulin E molecules. Fc epsilon RI aggregation by antigen induces activation of several down-stream signaling pathways, including LYN proto-oncogene, Src family tyrosine kinase, $S Y K$, linker for activation of $\mathrm{T}$ cells, extracellular signal related kinases, c-Jun $\mathrm{N}$-terminal kinases and p38 MAP kinase cascades, resulting in activation of the immune response (41). The involvement of the immune response in treatment with cyclophosphamide has been previously reported. Cyclophosphamide possesses dual anti-cancer and immunosuppressive properties and so it is also used in a variety of autoimmune disorders to inhibit graft rejection and graft-versus-host disease (17). When compared with sensitive CML cells, over-representation of immune response in resistant cells suggested its potential involvement in cyclophosphamide resistance. Other enrichment pathways, which at first appear irrelevant to cyclophosphamide, may also have a function in cyclophosphamide resistance. This requires further investigation.

Further signal transduction network analysis revealed that multiple genes were involved in significant biological processes and pathways. Notably, STAT5A, FYN and SYK, identified from biological process and KEGG pathway analysis, were the primary genes with the highest frequency at the center of the network. STAT5A is a member of the signal transducers and activators of transcription (STAT) family that is located in the cytoplasm and activated by a variety of cytokines. STATs are hypothesized to be important in multiple signaling pathways and thus involved in a number of cellular processes, including cell survival, proliferation, angiogenesis and immune evasion. They are frequently over-activated in solid tumor and blood malignancies. In particular, STAT5A and STAT5B are directly activated by oncogenic BCR-ABL tyrosine kinase $(42,43)$. 
Previous studies have demonstrated that inhibition of STAT5A restored the sensitivity of colorectal cancer cells to the cytotoxic drugs cisplatin and 5-fluorouracil (44). Therefore, the high levels of STAT5A observed in the present study may be involved in cyclophosphamide resistance in CML cells. FYN is an Src family kinase and a non-receptor tyrosine kinase. Therefore, $F Y N$ is able to phosphorylate tyrosine residues on key targets associated with multiple signaling pathways. The initial biological functions of $F Y N$ are focused on immune and neurological function. However, $F Y N$ is also involved in cell cycle, growth, proliferation, cell-cell adhesion and cell migration. FYN is overexpressed in a variety of cancers, including glioblastoma, melanoma and prostate cancer. Dasatinib is known to inhibit $F Y N$ activation and has been tested in pre-clinical and clinical trials for cancer treatment (45). A previous study has demonstrated the involvement of FYN in tamoxifen resistance in breast tumors. Overexpression of $F Y N$ in tamoxifen-sensitive cells impaired sensitivity in response to tamoxifen treatment (46). In addition, siRNA-mediated knockdown of FYN restored sensitivity to imatinib, a tyrosine kinase inhibitor, in CML cells (47). These reports supported the results of the present study, that highlighted FYN as one of the targets responsible for cyclophosphamide resistance. $S Y K$ is also a non-receptor tyrosine kinase, and is involved in intracellular signal transduction processes. It is important for immune and inflammatory responses, as well as cell survival in multiple cancers. $S Y K$ has been considered an interesting molecular target, and development of $S Y K$ inhibitors has been used to treat these pathologies (48). There remain no reports confirming the involvement of $S Y K$ in cyclophosphamide resistance, but $S Y K$ has been demonstrated to be associated with nilotinib resistance in CML cells (49). This network analysis provides valuable information on several potential genes that may be involved in cyclophosphamide resistance.

In addition, 5 genes were selected to validate their mRNA expression levels in 2 human CML cell lines (K-562 and KU812). The expression levels of the 5 genes were in line with those from the microarray experiments in the present study, including SYK, ALDH2, MDK, STAT5A and S100A4, further confirming the important association between expression of these genes and drug resistance.

To further evaluate whether increased levels of these genes was functionally important for cyclophosphamide resistance, siRNA was used to inhibit the expression of two highly expressed genes, STAT5A and S100A4, in K-562 cells. The survival of cells following treatment with 4-HC was subsequently determined. The results demonstrated that $4-\mathrm{HC}$ significantly decreased cell survival in the siSTAT5A and siS100A4 group, while the siControl group exhibited relative resistance to 4-HC treatment. Therefore, high expression of STAT5A or S100A4 in CML cells was important for cyclophosphamide resistance.

In conclusion, microarray technologies were used to comprehensively analyze simultaneous changes in gene expression between cyclophosphamide-sensitive and resistant CML cells, and several potential genes related with cyclophosphamide resistance were identified. Further functional validations were also performed, and revealed that STAT5A and S100A4 conferred resistance to cyclophosphamide to $\mathrm{CML}$ cells. These findings provide novel insights into cyclophosphamide resistance in CML cells. The genes identified in the present study may also help to predict resistant mechanisms for other drugs, as biomarkers. It may be possible to use inhibitors of resistance genes in combination with drugs to enhance therapeutic efficacy in the future, resulting in improved chemotherapy strategies for patients with cancer.

\section{References}

1. Jemal A, Siegel R, Xu J and Ward E: Cancer Statistics, 2010. CA Cancer J Clin 60: 277-300, 2010

2. Ramchandren R and Schiffer C: Dasatinib in the treatment of imatinib refractory chronic myeloid leukemia. Biologics 3 : 205-214, 2009.

3. Sawyers CL: Chronic Myeloid Leukemia. N Engl J Med 340: 1330-1340, 1999

4. Jabbour E, Cortes J and Kantarjian H: Treatment selection after imatinib resistance in chronic myeloid leukemia. Target Oncol 4: 3-10, 2009

5. Nowell PC and Hungerford DA: A minute chromosome in human chronic granulocytic leukemia. Science 142: 1497, 1960.

6. Rowley JD: Letter: A new consistent chromosomal abnormality in chronic myelogenous leukaemia identified by quinacrine fluorescence and Giemsa staining. Nature 243: 290-293, 1973.

7. Mandanas R, Leibowitz D, Gharehbaghi K, Tauchi T, Burgess G, Miyazawa K, Jayaram H and Boswell H: Role of p21 RAS in p210 bcr-abl transformation of murine myeloid cells 82: 1838-1847, 1993.

8. Okuda K, Matulonis U, Salgia R, Kanakura Y, Druker B and Griffin J: Factor independence of human myeloid leukemia cell lines is associated with increased phosphorylation of the proto-oncogene Raf-1. Exp Hematol 22: 1111-1117, 1994.

9. Raitano AB, Halpern JR, Hambuch TM and Sawyers CL: The Bcr-Abl leukemia oncogene activates Jun kinase and requires Jun for transformation. Proc Natl Acad Sci USA 92: 11746-11750, 1995.

10. Sawyers CL, Callahan W and Witte ON: Dominant negative MYC blocks transformation by ABL oncogenes. Cell 70: 901-910, 1992.

11. Shuai K, Halpern J, ten Hoeve J, Rao X and Sawyers CL: Constitutive activation of STAT5 by the BCR-ABL oncogene in chronic myelogenous leukemia. Oncogene 13: 247-254, 1996.

12. Tan J, Cang S, Seiter K, Primanneni S, Ahmed N, Mathews T and Liu D: $\mathrm{t}(3 ; 9 ; 22) 3$-way chromosome translocation in chronic myeloid leukemia is associated with poor prognosis. Cancer Invest 27: 718-722, 2009.

13. Quintás-Cardama A and Cortes JE: Chronic myeloid leukemia: Diagnosis and treatment. Mayo Clin Proc 81: 973-988, 2006.

14. Jabbour E and Kantarjian H: Chronic myeloid leukemia: 2014 update on diagnosis, monitoring, and management. Am J Hematol 89: 547-556, 2014.

15. Angstreich GR, Smith BD and Jones RJ: Treatment options for chronic myeloid leukemia: Imatinib versus interferon versus allogeneic transplant. Curr Opin Oncol 16: 95-99, 2004.

16. Gora-Tybor J and Robak T: Targeted drugs in chronic myeloid leukemia. Curr Med Chem 15: 3036-3051, 2008.

17. Emadi A, Jones RJ and Brodsky RA: Cyclophosphamide and cancer: Golden anniversary. Nat Rev Clin Oncol 6: 638-647, 2009.

18. Wu J and Gentry R: gcrma: Background adjustment using sequence information. $\mathrm{R}$ package version 2.46.0.

19. Gentleman R, Carey V, Huber W and Hahne F: genefilter: genefilter: Methods for filtering genes from microarray experiments. $\mathrm{R}$ package version 1.56.0.

20. Smyth GK: Limma: Linear models for microarray data. In: Gentleman R, Carey V, Dudoit S, Irizarry R, Huber W (eds). Bioinformatics and Computational Biology Solutions Using R and Bioconductor. Springer, New York, pp397-420, 2005.

21. Tavazoie S, Hughes JD, Campbell MJ, Cho RJ and Church GM: Systematic determination of genetic network architecture. Nat Genet 22: 281-285, 1999.

22. Carlson M: GO.db: A set of annotation maps describing the entire Gene Ontology. R package version 3.0.0.

23. Carlson M: KEGG.db: A set of annotation maps for KEGG. R package version 3.2.3.

24. Tenenbaum D: KEGGREST: Client-side REST access to KEGG. $\mathrm{R}$ package version 1.14 .1 . 
25. Keshava Prasad TS, Goel R, Kandasamy K, Keerthikumar S, Kumar S, Mathivanan S, Telikicherla D, Raju R, Shafreen B, Venugopal A, et al: Human protein reference database-2009 update. Nucleic Acids Res 37 (Database Issue): D767-D772, 2009.

26. Chatr-aryamontri A, Breitkreutz BJ, Heinicke S, Boucher L, Winter A, Stark C, Nixon J, Ramage L, Kolas N, O'Donnell L, et al: The BioGRID interaction database: 2013 update. Nucleic Acids Res 41 (Database Issue): D816-D823, 2013.

27. McDowall MD, Scott MS and Barton GJ: PIPs: Human protein-protein interaction prediction database. Nucleic Acids Res 37 (Database Issue): D651-D656, 2009.

28. Smoot ME, Ono K, Ruscheinski J, Wang PL and Ideker T: Cytoscape 2.8: New features for data integration and network visualization. Bioinformatics 27: 431-432, 2011.

29. Livak KJ and Schmittgen TD: Analysis of relative gene expression data using real-time quantitative PCR and the 2(-Delta Delta C(T)) Method. Methods 25: 402-408, 2001.

30. Colvin O: An overview of cyclophosphamide development and clinical applications. Curr Pharm Des 5: 555-560, 1999.

31. Sládek NE: Leukemic cell insensitivity to cyclophosphamide and other oxazaphosphorines mediated by aldehyde dehydrogenase(s). In: Clinically Relevant Resistance in Cancer Chemotherapy. Springer, pp161-175, 2002.

32. Russo JE, Hilton J and Colvin OM: The role of aldehyde dehydrogenase isozymes in cellular resistance to the alkylating agent cyclophosphamide. Prog Clin Biol Res 290: 65-79, 1989.

33. Moreb J, Muhoczy D, Ostmark B and Zucali J: RNAi-mediated knockdown of aldehyde dehydrogenase class-1A1 and class-3A1 is specific and reveals that each contributes equally to the resistance against 4-hydroperoxycyclophosphamide. Cancer Chemother Pharmacol 59: 127-136, 2007.

34. Plebuch M, Soldan M, Hungerer C, Koch L and Maser E: Increased resistance of tumor cells to daunorubicin after transfection of cDNAs coding for anthracycline inactivating enzymes. Cancer Lett 255: 49-56, 2007.

35. Kadomatsu K and Muramatsu T: Midkine and pleiotrophin in neural development and cancer. Cancer Lett 204: 127-143, 2004.

36. Kang HC, Kim IJ, Park HW, Jang SG, Ahn SA, Yoon SN, Chang HJ, Yoo BC and Park JG: Regulation of MDK expression in human cancer cells modulates sensitivities to various anticancer drugs: MDK overexpression confers to a multi-drug resistance. Cancer Lett 247: 40-47, 2007.

37. Xu YY, Mao XY, Song YX, Zhao F, Wang ZN, Zhang WX, $\mathrm{Xu} \mathrm{HM}$ and Jin F: Midkine confers Adriamycin resistance in human gastric cancer cells. Tumor Biol 33: 1543-1548, 2012.

38. Mishra S, Siddique H and Saleem M: S100A4 calcium-binding protein is key player in tumor progression and metastasis: Preclinical and clinical evidence. Cancer Metastasis Rev 31: $163-172,2012$
39. Mahon PC, Baril P, Bhakta V, Chelala C, Caulee K, Harada T and Lemoine NR: S100A4 Contributes to the suppression of BNIP3 expression, chemoresistance and inhibition of apoptosis in pancreatic cancer. Cancer Res 67: 6786-6795, 2007.

40. Hekmat O, Munk S, Fogh L, Yadav R, Francavilla C, Horn H, Würtz SØ, Schrohl AS, Damsgaard B, Rømer MU, et al: TIMP-1 increases expression and phosphorylation of proteins associated with drug resistance in breast cancer cells. J Proteome Res 12: 4136-4151, 2013.

41. Rivera J and Olivera A: A current understanding of Fc epsilon RI-dependent mast cell activation. Curr Allergy Asthma Rep 8: 14-20, 2008.

42. Yu H and Jove R: The STATs of cancer-new molecular targets come of age. Nat Rev Cancer 4: 97-105, 2004.

43. Schaller-Schönitz M, Barzan D, Williamson AJ, Griffiths JR, Dallmann I, Battmer K, Ganser A, Whetton AD, Scherr M and Eder M: BCR-ABL Affects STAT5A and STAT5B differentially. PLoS One 9: e97243, 2014.

44. Hong X, Chen G, Wang M, Lou C, Mao Y, Li Z and Zhang Y: STAT5a-targeting miRNA enhances chemosensitivity to cisplatin and 5-fluorouracil in human colorectal cancer cells. Mol Med Rep 5: 1215-1219, 2012.

45. Saito YD, Jensen AR, Salgia R and Posadas EM: Fyn: A novel molecular target in cancer. Cancer 116: 1629-1637, 2010.

46. Elias D, Vever H, Lankholm AV, Gjerstorff MF, Yde CW, Lykkesfeldt AE and Ditzel HJ: Gene expression profiling identifies FYN as an important molecule in tamoxifen resistance and a predictor of early recurrence in patients treated with endocrine therapy. Oncogene 34: 1919-1927, 2015.

47. Fenouille N, Puissant A, Dufies M, Robert G, Jacquel A, Ohanna M, Deckert M, Pasquet JM, Mahon FX, Cassuto JP, et al: Persistent activation of the Fyn/ERK kinase signaling axis mediates imatinib resistance in chronic myelogenous leukemia cells through upregulation of intracellular SPARC. Cancer Res 70: 9659-9670, 2010

48. Riccaboni M, Bianchi I and Petrillo P: Spleen tyrosine kinases: Biology, therapeutic targets and drugs. Drug Discov Today 15: 517-530, 2010.

49. Gioia R, Leroy C, Drullion C, Lagarde V, Etienne G, Dulucq S, Lippert E, Roche S, Mahon FX and Pasquet JM: Quantitative phosphoproteomics revealed interplay between Syk and Lyn in the resistance to nilotinib in chronic myeloid leukemia cells. Blood 118: 2211-2221, 2011. 